\title{
Assessing momentary states of relaxation: The Relaxation State Questionnaire
}

Sarah Steghaus \& Christian H. Poth

Neuro-cognitive Psychology and Center of Cognitive Interaction Technology, Bielefeld University

Corresponding Author:

Sarah Steghaus

Department of Psychology

Bielefeld University, P. O. Box 100131

D-33501 Bielefeld

Germany

sarah.steghaus@uni-bielefeld.de 


\begin{abstract}
Stress is ubiquitous in everyday life and hazardous for mental and physical health. To prevent or ameliorate stress-related disease, approaches such as relaxation exercises aim to counteract stress by inducing short-lasting states of relaxation. Critically, current assessments capture the mid-and long-term consequences of relaxation but are unable to quantify these states of relaxation. To address this problem, we developed the Relaxation State Questionnaire (RSQ). We assessed the item properties, reliability, and validity of the questionnaire in an online study. Construct validity was examined through correlations with the Perceived Stress Questionnaire (PSQ; Fliege et al., 2005). An exploratory factor analysis revealed four factors capturing the momentary state of muscle tension, sleepiness, cardiovascular activity, and general relaxation. The items had high loadings on their factors and the reliability and construct validity of the RSQ were both high. These findings establish the RSQ as a tool to measure states of relaxation. As such, the RSQ opens up investigations of the immediate effects and effectiveness of relaxation exercises.
\end{abstract}




\section{Introduction}

Stress is a major risk factor for serious health problems, such as cardiovascular disease, diabetes, mental disorders such as anxiety and depression, and several other illnesses (U.S. Department of Health and Human Services, National Institutes of Health, National Institute of Mental Health, 2019). A vast number of different treatments have been developed to manage and reduce stress. The most prominent treatments may broadly be classified as relaxation activities. To assess the effectiveness of stress management, one needs measures of subjective stress and relaxation. These exist only for long-term relaxation effects and perceived stress, but there are no adequate measures for shortterm relaxation effects. This is surprising because any immediate help provided by relaxation techniques is left uninvestigated. Here, we provide a new questionnaire, the Relaxation State Questionnaire (RSQ) to solve this problem. We show that the RSQ captures momentary states of relaxation efficiently, reliably, and validly. Thus, the RSQ paves the way for research on the shortterm effects and effectiveness of relaxation exercises and interventions.

\section{Stress and Short-Term Relaxation}

Stress is ubiquitous in everyday life: From financial stress, to stress with friends and family, to work stress and environmental stress (for an overview, see Wadhwa, 2017). As diverse as the sources of stress can be are its consequences. For instance, stress hinders the immune system (Herbert \& Cohen, 1993), poses a risk for diabetes (Lloyd et al., 2005), causes depression (Tennant, 2002), and is associated with a number of other diseases (U.S. Department of Health and Human Services, National Institutes of Health, National Institute of Mental Health, 2019). Throughout history, relaxation exercises have been developed as tools to relieve stress and prevent its negative ramifications (Wadhwa, 2017). Current exercises range from breathing exercises to progressive muscle relaxation (PMR). To date, a number of studies delivered evidence that relaxation exercises such as PMR (Chaudhuri et al., 2014), Tai-chi (Wang et al., 2010), or guided imagery and hypnosis (Gruzelier, 2002) effectively reduce stress.

To benefit from relaxation exercises, one does not need years of experience and training. For example, 1 hour of daily relaxation practice for 6 weeks can lead to a reduction of heart rate and blood pressure in students (Pal et al., 2014). Furthermore, it was shown that relaxation moderated the relationship between occupational stress and mental health symptoms one month later (Sawhney et al., 2018). In another study, the effects of only 3 consecutive days of mindfulness mediation were compared to sham meditation and a control group (Zeidan et al., 2010). The authors found that the meditation exercise increased the mood and decreased both depression and heart rate. One study could even demonstrate positive effects of relaxation exercises (PMR and meditation) on anxiety measures after just one session (Rausch et al., 2006). 


\section{Measuring Short-Term Relaxation}

However, to directly assess the current state of relaxation (or consequently stress) from the participants' point of view, a suitable questionnaire is needed. Several relaxation questionnaires already exist, for example, the Relaxation Inventory by Crist et al. (1989) or the Smith Relaxation States Inventory- Revised (SRSI-R; Smith, 2007). These questionnaires, however, measure relaxation as a broad construct and more as a trait than as a state. For example, one item of the Relaxation Inventory is "I am worried about how much money I have.". Items such as these refer to mental states over longer periods and thus cannot capture the momentary changes in the state of relaxation. Also, the SRSI-R covers the dimension "Mystery", referring to "feeling the deeper mystery of things beyond one's understanding" (Smith et al., 2000, p. 1202), which also does not have a clear face validity regarding changes in relaxation states as they are necessary to evaluate relaxation techniques. Hites and Lundervold (2013) investigated the relationship between both questionnaires and a physiological observation of relaxation (including assessment of breathing and different body areas and muscles) and could not find the desired correlations. Thus, in sum, current tools are unable to assess the momentary states of relaxation, which means that questions of the immediate effects of relaxation exercises still remain unanswerable.

\section{The Relaxation State Questionnaire}

To address these problems, we developed a new questionnaire, the Relaxation State Questionnaire (RSQ), which assesses a person's momentary state of relaxation. Since it is specifically tailored to measure short-term relaxation states, the RSQ can be used to assess the immediate effectiveness of relaxation exercises (e.g., by comparing measures before and after a relaxation exercise).

Here, we introduced the RSQ with its 10 items, and investigated its psychometric properties, such as its item properties (mean values, item difficulties, and item discrimination), its factorial validity (using exploratory factor analysis) and reliability. In addition, we validated the RSQ by obtaining correlations with specific subscales from an instrument capturing perceived stress (the German version of the Perceived Stress Questionnaire Fliege et al., 2005). That is, we assessed the scales "joy" and "tension", whose items reflect some mental aspects of relaxation and the opposite state of relaxation, respectively. Thus, we predicted positive correlations of RSQ scales capturing relaxation with the joy scale, and negative correlations with the tension scale. 


\section{Method}

\section{Preregistration \& Power Analysis}

We preregistered our study to the Open Science Framework (OSF;

https://osf.io/syext/?view only=ce0d062f1cd24375ad4be13ab3a6c310). Furthermore, we conducted an a priori power analysis for the correlations and the exploratory factor analysis (EFA). A power analysis with the software G*Power (Faul et al., 2007, version 3.1) showed that for a power of .80 , an alpha error probability of .05 , and an effect size of .40 (intermediate effect) for the correlations, a sample size of 38 participants would be needed. For smaller correlations of around .30, 67 participants would be necessary, and for correlations of .20, 153 participants would be needed.

The number of participants for the exploratory factor analysis depends on the number of factors of the questionnaire, the factor loadings and the number of items loading on each factor (de Winter et al., 2009). Since all these parameters had yet to be determined for our questionnaire, the required minimum sample size could vary between 12 and 120 participants (de Winter et al., 2009, p. 154 ff.).

Based on the power analyses and because our data collection had to take place within a time of 3 weeks, we planned and preregistered a sample size of minimally 40 and maximally 120 participants.

\section{Sample}

In total, 98 participants were recruited for the experiment. Psychology students at Bielefeld University were recruited through the Psychology Department's participant pool) and received course credit for participation. Furthermore, the study was distributed via a snowball system and Facebook groups to also obtain data from non-students.

Participants who did not report an age of at least 18 years, who were not at least "almost fluent" in German, and who indicated afterwards that they did not read and answer conscientiously, but merely clicked through, were excluded from the data set. Therefore, the final sample consisted of 92 participants (77 females, 14 males, and 1 non-binary).

The mean age was 28.4 years $(S D=13.9 ; M d n=23)$ with a range from 18 to 71 years. For their occupational status, 73 participants stated they were students, 10 employed, 2 job-seeking, and 6 participants stated they were retired.

All participants gave informed consent before the study. The experiment conformed to the ethical guidelines of the German Psychological Association (DGPs) and was approved by the ethics committee of Bielefeld University. 


\section{Measures}

\section{Relaxation State Questionnaire - RSQ}

The goal was to develop a questionnaire that assesses the current relaxation state of a person. Since the questionnaire is supposed to be used in future studies and PMR experiments, the main goal was to assess the relaxational state of the body and mind with a focus on the muscles. Out of a review of different existing measurements (Crist et al., 1989; Sakakibara et al., 2014; Smith et al., 2000), 10 items emerged that seem to fit this purpose. Table 1 displays the items and their English translation. The first five items were thought to cover the physical component of the relaxed state. For example, Question 4 states "My muscles feel relaxed.". Three of the five questions specifically focus on the muscles, the other two on the breathing and the heart rate, which are two reliable indices for the level of stress hormones (Wadhwa, 2017, Chapter 9). Items 6-10 focus on the components of mental relaxation. For example, Item 7 states "I am completely calm right now". Along with the 10 items, participants received the following instruction: "Below you will find a series of statements. Please read each statement and choose from the five answers the one that best describes, how much the statement is accurate to your feelings and condition right now, in this moment. There are no right or wrong answers. Please do not think to long about each statement and do not omit one." (Instructions translated from German, the original instruction can be found in Appendix A) The five response categories were: not correct at all - rather not correct - neither nor rather correct - entirely correct.

\section{Perceived Stress Questionnaire - PSQ}

To validate the new questionnaire, the German version of the Perceived Stress Questionnaire (PSQ) by Fliege et al. (2005) was used. The questionnaire "assesses subjectively experienced stress independent of a specific and objective occasion" (p. 78) and has a Cronbach's Alpha of .92 in a student population. Since the questionnaire targets "different facets of perceived stress" (p. 79) that are not all relevant in this context, only the subscales "joy" and "tension" were used. The scale tension "explores tense disquietude, exhaustion, and the lack of relaxation" (p. 83). Therefore, it should correlate negative with a higher score of relaxation of the RSQ. The joy subscale "is concerned with positive feelings of challenge, joy, energy, and security" (p. 83). Like the RSQ, each item is formulated as a statement (e.g., "I'm feeling tense.") and participants are asked to rate on a fourpoint scale (almost never - sometimes - often - usually) how accurately the statement describes them, concerning the last two weeks of their lives. 


\section{Table 1}

Items and Their English Translation
Item
Items in German
English Translation

$1 \quad$ Mein Atem ist schneller als gewöhnlich. (R) My breathing is faster than usual. (R)

\begin{tabular}{cll}
\hline 2 & Mein Herz schlägt schneller als sonst. (R) & My heart is beating faster than usual. (R) \\
\hline 3 & Meine Muskeln fühlen sich angespannt & My muscles feel tense and cramped \\
& und verkrampft an (Hand zur Faust geballt; & (clenched fist and/or jaw; furrowed \\
& Kiefer angespannt; gerunzelte Stirn). (R) & brow). (R) \\
\hline 4 & Meine Muskeln fühlen sich entspannt an. & My muscles feel relaxed. \\
\hline 6 & Meine Muskeln fühlen sich locker an. & My muscles feel loose. \\
\hline 7 & Ich fühle mich sehr entspannt. & I'm feeling very relaxed. \\
\hline 8 & Ich fühle mich schläfrig und müde. & Right now, I am completely calm. \\
\hline 9 & Ich bin kurz davor einzunicken. & I'm feeling sleepy and tired. \\
\hline 10 & Ich fühle mich erfrischt und wach. (R) & I'm feeling refreshed and awake. (R) \\
\hline
\end{tabular}

Note. Reverse-coded items are indicated by (R).

\section{Design and Procedure}

The online study took about $5-15$ minutes to complete; however, participants had no time limit for the completion of the questions. After giving informed consent, the RSQ and the two subscales of the PSQ were presented to the participants in a random order. The order of items within each questionnaire was also randomized. Every item had to be answered, otherwise participants were not able to continue with the questionnaire and complete the study. At the end, participants were asked for demographic information. The detailed procedure and phrasing of the study can be found in the study documentation on the OSF

(https://osf.io/syext/?view only=ce0d062f1cd24375ad4be13ab3a6c310). 


\section{Data Analyses}

Statistical analyses were performed in R (R Core Team, 2020; version 4.0.2). A commented RScript with all analyses can also be found on the OSF (https://osf.io/syext/?view only=ce0d062f1cd24375ad4be13ab3a6c310).

Inversely coded items were recoded with the recode function of the car package (Fox \& Weisberg, 2019). The Kaiser-Meyer-Olkin measure of sampling adequacy (Kaiser, 1970) and the Bartlett's test of sphericity (Bartlett, 1950) were computed to determine whether it was appropriated to perform a factor analysis on our data (Dziuban \& Shirkey, 1974). Item analysis and exploratory factor analysis were conducted with the describe function of the R-package psych (Revelle, 2020). For the item difficulty, the popularity index according to Dahl was calculated (Moosbrugger \& Kelava, 2012, p. 81). Item discrimination was determined with the alpha function of the psych package. Furthermore, to compute the reliability, the measurement model of the items must be determined first (see Eid et al., 2017 , p. 851 f.). Only then the reliability can be computed accurately. This was done with the lavaan package (Rosseel, 2012). Reliability was then computed with the omega.tot function, using the Lambda4 and GPArotation packages (Bernaards \& Jennrich, 2005; Hunt, 2013). Correlations between PSQ and RSQ were computed using the stats package (R Core Team, 2020). Also, plots for the correlations were examined using the plot function to detect non-linear relations and statistical outliers.

\section{Results}

\section{Exploratory Factor Analysis}

Before computing the exploratory factor analysis, both, the Kaiser-Meyer-Olkin (KMO) measure, and the Bartlett test of sphericity were computed. The overall quality of the correlation matrix was .73 and thus above the cutoff of .5 proposed by Kaiser and Rice (1974). The Bartlett test of sphericity was significant $(p<.001)$ and therefore justified a dimension reducing procedure.

An exploratory factor analysis of all 10 items was then performed in R. Since we expected factors to be correlated, an oblique rotation (promax) was conducted. The factors were interpreted based on the factor pattern matrix. This yielded a four-factor solution with three or two items loading on one factor, respectively. The factor loadings ranged between 0.44 and 1.07 and no cross loadings over 0.40 were found (see Table 2).

Table 3 shows the correlations between the four found factors. Three factors (1, 3, and 4$)$ were moderately positive correlated, Factor 2 (sleepiness score), however, correlated negatively with the others. 


\section{Table 2}

Results From the Factor Analysis of the RSQ

\begin{tabular}{|c|c|c|c|c|}
\hline \multirow[t]{2}{*}{ Item } & \multicolumn{4}{|c|}{ Factor loading } \\
\hline & 1 & 2 & 3 & 4 \\
\hline \multicolumn{5}{|l|}{ Factor 1: Muscle Score } \\
\hline 5. My muscles feel loose. & 0.93 & 0.13 & -0.10 & 0.08 \\
\hline 4. My muscles feel relaxed. & 0.86 & 0.00 & -0.08 & 0.07 \\
\hline $\begin{array}{l}\text { 3. My muscles feel tense and cramped } \\
\text { (clenched fist and/or jaw; furrowed brow). (R) }\end{array}$ & 0.53 & -0.06 & 0.05 & 0.09 \\
\hline \multicolumn{5}{|l|}{ Factor 2: Sleepiness Score } \\
\hline 8. I'm feeling sleepy and tired. & 0.07 & 1.07 & 0.20 & -0.03 \\
\hline 10. I'm feeling refreshed and awake. (R) & 0.16 & 0.75 & 0.00 & -0.29 \\
\hline 9. I'm about to doze off. & -0.19 & 0.44 & -0.17 & 0.28 \\
\hline \multicolumn{5}{|l|}{ Factor 3: Cardiovascular Score } \\
\hline 1. My breathing is faster than usual. (R) & -0.09 & 0.11 & 0.89 & -0.02 \\
\hline 2. My heart is beating faster than usual. (R) & -0.02 & -0.01 & 0.71 & 0.11 \\
\hline \multicolumn{5}{|l|}{ Factor 4: General Relaxation Score } \\
\hline 6. I'm feeling very relaxed. & 0.15 & -0.01 & -0.06 & 0.94 \\
\hline 7. Right now, I am completely calm. & 0.14 & -0.04 & 0.21 & 0.56 \\
\hline
\end{tabular}

Note. The extraction method was principal axis factoring with an oblique rotation (promax with Kaiser normalization). Factor loadings above 0.40 are in bold. Reverse-scored items are denoted with (R). 


\section{Table 3}

Factor Correlations for the Four Factors

\begin{tabular}{lllll}
\hline \multicolumn{1}{c}{ Factor } & 1 & 2 & 3 & 4 \\
1. Muscle Score & - & -.42 & .38 & .43 \\
2. Sleepiness Score & & - & -.49 & -.24 \\
3. Cardiovascular Score & & - & .32 \\
4. General Relaxation Score & & & - \\
\hline
\end{tabular}

\section{Item Analyses}

For the item analyses, means, standard deviations, and item difficulty were computed for all items. Furthermore, we computed the item's discrimination power (using the correlation between the item score and the rest score of the scale) for those items who loaded on a factor with more than two items. All item properties can be found in Table 4. 


\section{Table 4}

Item Properties of the 10 Items of the RSQ

\begin{tabular}{|c|c|c|c|c|}
\hline Item & $M$ & $S D$ & $P$ & $r t$ \\
\hline My breathing is faster than usual. (R) & 4.14 & 0.93 & 78.53 & \\
\hline My heart is beating faster than usual. $(R)$ & 4.02 & 0.96 & 75.54 & \\
\hline $\begin{array}{l}\text { My muscles feel tense and cramped } \\
\text { (clenched fist and/or jaw; furrowed brow). (R) }\end{array}$ & 3.62 & 1.11 & 65.49 & .56 \\
\hline My muscles feel relaxed. & 3.33 & 1.06 & 58.15 & .74 \\
\hline My muscles feel loose. & 3.30 & 1.16 & 57.61 & .73 \\
\hline I'm feeling very relaxed. & 2.91 & 1.08 & 47.83 & \\
\hline Right now, I am completely calm. & 3.32 & 1.09 & 57.88 & \\
\hline I'm feeling sleepy and tired. & 3.13 & 1.18 & 53.26 & .72 \\
\hline I'm about to doze off. & 2.09 & 1.14 & 27.17 & .44 \\
\hline I'm feeling refreshed and awake. (R) & 3.33 & 1.03 & 58.15 & .62 \\
\hline
\end{tabular}

\section{Measurement Model and Reliability}

To adequately compute reliability, we tested the model fit of different measurement models by comparing the variance/covariance matrix of the four subscales of our data with the model implied matrices (Eid et al., 2017, p. 855; Graham, 2006).

We first tested the tau congeneric model. The tau congeneric model is the least restrictive measurement model, allowing different means, variances, and covariances within the matrix. This model simply assumes that the items of one subscale depend on the same latent variable, not necessarily to the same extent. The tau congeneric model had an overall acceptable fit. Fit indices can be found in Table 5 . 


\section{Table 5}

Fit Indices for Both Measurement Models

\begin{tabular}{|c|c|c|}
\hline \multirow[t]{2}{*}{ Fit Indices } & \multicolumn{2}{|c|}{ Model } \\
\hline & Tau congeneric & Essential tau equivalent \\
\hline$\chi^{2} / \mathrm{df}$ & 1.7 & 2 \\
\hline CFI & .95 & .91 \\
\hline RMSEA & $.09[.04-.13]$ & $.1[.07-.14]$ \\
\hline SRMR & .06 & .1 \\
\hline AIC & 2408.38 & 2417.07 \\
\hline
\end{tabular}

The next-restrictive measurement model to compare the tau congeneric model to, is the essentially tau equivalent model. In the essentially tau equivalent model, the additional assumption that the covariances of the matrix are the same is implemented. This means, that the items of one subscale depend on the same latent variable to the same extend. The model fit got worse for this model in comparison to the tau congeneric model (see Table 5). Hence, because of its better fit, we chose the tau congeneric model for our data. Therefore, we computed Omega as a measurement for reliability, as proposed by Eid et al. (2017, p. 869; for a discussion of the different parameters see also Green \& Yang, 2015).

Reliability for the whole questionnaire was $\omega=0.83$. For the muscle score, reliability was $\omega=0.83$, for the sleepiness score $\omega=0.79$. Since the reliability can only be computed for subscale with more than two items, it could not be computed for the cardiovascular score and the general relaxation score. All computed reliability scores are above the cut-off values proposed for example by Reise and colleagues (2013) or Catalán (2019). Therefore, the reliability of the questionnaire can be rated as high.

\section{Correlations with PSQ}

To examine the construct validity, first the total score for both the joy and the tension scale of the PSQ were computed according to the formula given by Fliege and colleagues (2005). Then, the scores were correlated with the four scales of the RSQ. No non-linear relations between the scores could be found when examining the plots. Table 6 shows the correlation between the RSQ and the PSQ scores. 
All correlations were significant and remained significant after adjusting for multiple testing with the Bonferroni-Holm correction (see Eid et al., 2017, p. 418). A table with $p$ values and significance levels after the correction can be found in Appendix B. Table 6 shows the correlation between the scales of the PSQ and the RSQ. As hypothesized the tension scale and the RSQ scores (except for the sleepiness score) correlate negatively. For the joy scale, the assumed positive correlations were found, again except for the sleepiness score. However, since the sleepiness score surprisingly correlated negative with the other scores of the RSQ in the EFA, we expected to find these reverse correlations (compared to the other RSQ scales) for the PSQ scales. Therefore, all correlations had the expected direction.

\section{Table 6}

Correlations Between RSQ and PSQ Scales

\begin{tabular}{lcc}
\hline \multicolumn{1}{c}{ RSQ Scales } & \multicolumn{2}{c}{ PSQ Scales } \\
\cline { 2 - 3 } Muscle score & Tension & $.25^{* *}$ \\
Sleepiness score & $-.43^{* * *}$ & $-.45^{* * *}$ \\
Cardiovascular score & $.46^{* * *}$ & $.24^{*}$ \\
General relaxation score & $-.20^{*}$ & $.22^{*}$ \\
\hline Note. All correlations were tested one-tailed. ${ }^{*} p<.05 . * * p<.01 . * * * p<.001$.
\end{tabular}

All correlations remained significant after Bonferroni-Holm correction.

\section{Discussion}

The aim of this study was to develop and validate a new questionnaire that asses the momentary state of relaxation, the Relaxation State Questionnaire (RSQ). The factorial validity showed a fourfactor solution for our 10 items. Item properties and reliability were also computed. To further validate the questionnaire, we correlated it with the two subscales "joy" and "tension" of the German version of the Perceived Stress Questionnaire. The results of our analyses showed that the RSQ is a reliable and valid instrument with good item properties. As such, the RSQ paves the way for research on momentary states of subjective relaxation and thus of the immediate effects and effectiveness of relaxation exercises. 


\section{Item Properties and Factory Validity}

The item properties of the questionnaire were all satisfactory. All items were used to their full range, except for item 2 ("My heart is beating faster than usual"), which only had a range from 2-5. Furthermore, no item displayed ceiling or floor effects. The item difficulty ranged from 47.83 to 78.53 , with the exception of item 9 ("I'm about to doze off."), whose difficulty was the lowest with 27.17. Therefore, all items have medium difficulties and are suited for the questionnaire (see e.g., Moosbrugger \& Kelava, 2012, p. 87).

The exploratory factor analysis proposed a four-factor solution for the items. With only 10 items in total, this means that there are two factors with only two items each. Even though this means that item discrimination and reliability could not be computed for these scales, all items had very high loadings on their respective factors ( 0.44 to 1.07 ) and no cross loadings above 0.4 were detected. This indicates that the items fit very well with their respective factors and other factor solutions would not seem appropriate. Even though, the four-factor solution was not expected beforehand, the different scores of the RSQ may be very useful to differentiate relaxation and effects of different relaxation exercises: The muscle score can be useful for PMR exercises, the cardiovascular score for breathing exercises, and the sleepiness score can function as a manipulation check to test whether exercises actually relaxed participants and patient or just made them drowsy.

Reliability and item discrimination were computed for the two scales that had more than two items. Omega reached 0.83 for the questionnaire, exceeding the requirements proposed by Catalán (2019) and thus revealing a reliable measurement. Item discrimination values ranged between .56 and .74, indicating that all items can adequately differentiate between participants with high and low levels of relaxation (cf. Moosbrugger \& Kelava, 2012, p. 86). Therefore, the item selection for the questionnaire succeeded and no items should be excluded.

\section{Construct Validity}

To further determine construct validity for our new questionnaire, correlations with the Perceived Stress Questionnaire (Fliege et al., 2005) were computed. Since the sleepiness score (Factor 2 ) is negatively correlated with the other factors, the four scales/factors were correlated independently with the two subscales of the PSQ. As hypothesized, there was a negative correlation between the first factor (containing the items for the muscles) and the scale "tension". This validates the intended objective for these items.

Also, the items correlated negative with the subscale "joy". As one would expect, the items on the sleepiness scale correlate negative with "tension" scale and negative with the "joy" scale. Construct validity of our questionnaire can therefore be assumed. 


\section{Assessing Momentary States of Subjective Relaxation}

To successfully measure momentary states and changes in the subjective feeling of relaxation, self-report measures, such as this questionnaire, are essential. They capture the phenomenal state of relaxation that is private and only (if at all) accessible by report. Also, self-reports are known to have a high accuracy, predictability, and utility (Eid \& Diener, 2006, Chapter 3). Indirect indicators of relaxation (e.g., physiological measures) cannot adequately represent the participants' state of mind and inner subjective emotional world. After all, the most direct way to assess relaxation is to ask if one feels relaxed. As such, even though one might argue that two items per scale in our questionnaire are too few, these items have the highest face validity (e.g., Item 6 "I'm feeling very relaxed.") and should thus be kept in the questionnaire. This is consistent with their high loadings on their respective factors.

Since completing a questionnaire is a cognitive activity which might interfere with the current state of relaxation, we aimed for a short and efficient, and yet reliable questionnaire. While the 10 items do cover several aspects of relaxation and are suited for various relaxation exercises, the overall time needed to complete the questionnaire is very short. In highly dynamic situations, for example, within cognitive experiments in which questionnaires would interrupt the task too much, the usage of only one or two score of the RSQ can even be considered. This procedure would allow a most efficient measurement of certain aspects of the relaxation state without inferring with the relaxation effect itself.

\section{Assessing the Effectiveness of Relaxation Exercises}

Currently, there are no reliable and valid tools for assessing momentary states of relaxation from the participant's point of view. As a consequence, short-term changes in these states cannot be assessed. This is a problem for the empirical evaluation of all relaxation exercises because their short-term effects and their effectiveness cannot be quantified. The RSQ fills this gap and provides a reliable and valid assessment of relaxation, which allows the evaluation of relaxation exercises such as progressive muscle relaxation (e.g., Borkovec \& Sides, 1979; McCallie et al., 2006), mindfulness meditation (e.g., Heppner \& Shirk, 2018; Mars \& Abbey, 2010), and other meditation techniques.

\section{Limitations}

One might ask if the sample sizes of this study was sufficient for our exploratory factor analysis. This seems to be the case, since it has been shown that sample sizes as ours can yield reliable results (Costello \& Osborne, 2005; de Winter et al., 2009; Kyriazos, 2018). This is also supported by the high factor loadings for the questionnaire, which indicate that only a smaller number of participants was 
needed for reliable results. Thus, taken together, our present results seem reliable and they establish the RSQ as a reliable and valid tool for assessing states of relaxation.

It is an open question how sensitive the RSQ is for manipulations of the relaxation state by interventions that target subjective relaxation. This question provides an interesting avenue for further studies that manipulate the state of relaxation experimentally and monitor the resulting change in relaxation. Thus, as opposed to assessing the effectiveness of a relaxation exercise, such experiments could in turn establish the capability of the RSQ to register predicted changes of relaxation caused by relaxation interventions.

\section{Conclusions}

We developed a questionnaire assessing the momentary state of subjective relaxation (Relaxation State Questionnaire, RSQ). Item properties, factor loadings, and reliability were all satisfactory. In addition, the construct validity could be established by means of correlation with an established instrument. With 10 items, the questionnaire is brief and can be easily implemented in a range of research settings. Taken together, the RSQ paves the way for research on short-lasting states of relaxation and opens up investigations of the immediate effectiveness of relaxation exercises. 


\section{References}

Bartlett, M. S. (1950). Tests of significance in factor analysis. British Journal of Statistical Psychology, 3(2), 77-85. https://doi.org/10.1111/j.2044-8317.1950.tb00285.x

Bernaards, C. A., \& Jennrich, R. I. (2005). Gradient projection algorithms and software for arbitrary rotation criteria in factor analysis. Educational and Psychological Measurement, 65, 676-696.

Borkovec, T. D., \& Sides, J. K. (1979). Critical procedural variables related to the physiological effects of progressive relaxation: A review. Behaviour Research and Therapy, 17(2), 119-125. https://doi.org/10.1016/0005-7967(79)90020-2

Catalán, H. E. N. (2019). Reliability, population classification and weighting in multidimensional poverty measurement: A Monte Carlo study. Social Indicators Research, 142(3), 887-910. https://doi.org/10.1007/s11205-018-1950-z

Costello, A. B., \& Osborne, J. (2005). Best practices in exploratory factor analysis: Four recommendations for getting the most from your analysis. Practical Assessment, Research, and Evaluation, 10(Article 7). https://doi.org/10.7275/JYJ1-4868

Crist, D. A., Rickard, H. C., Prentice-Dunn, S., \& Barker, H. R. (1989). The Relaxation Inventory: Selfreport scales of relaxation training effects. Journal of Personality Assessment, 53(4), 716726. https://doi.org/10.1207/s15327752jpa5304_8

de Winter, J. C. F., Dodou, D., \& Wieringa, P. A. (2009). Exploratory factor analysis with small sample sizes. Multivariate Behavioral Research, 44(2), 147-181. https://doi.org/10.1080/00273170902794206

Dziuban, C. D., \& Shirkey, E. C. (1974). When is a correlation matrix appropriate for factor analysis? Some decision rules. Psychological Bulletin, 81(6), 358-361. https://doi.org/10.1037/h0036316

Eid, M., \& Diener, E. (Eds.). (2006). Handbook of multimethod measurement in psychology (1st ed). American Psychological Association.

Eid, M., Gollwitzer, M., \& Schmitt, M. (2017). Statistik und Forschungsmethoden [Statistics and research methods] (5., korrigierte Auflage). Beltz. 
Faul, F., Erdfelder, E., Lang, A.-G., \& Buchner, A. (2007). G*Power 3: A flexible statistical power analysis program for the social, behavioral, and biomedical sciences. Behavior Research Methods, 39(2), 175-191. https://doi.org/10.3758/BF03193146

Fliege, H., Rose, M., Arck, P., Walter, O. B., Kocalevent, R.-D., Weber, C., \& Klapp, B. F. (2005). The Perceived Stress Questionnaire (PSQ) reconsidered: Validation and reference values from different clinical and healthy adult samples. Psychosomatic Medicine, 67(1), 78-88. https://doi.org/10.1097/01.psy.0000151491.80178.78

Fox, J., \& Weisberg, S. (2019). An R companion to applied regression (Third). Sage. https://socialsciences.mcmaster.ca/jfox/Books/Companion/

Graham, J. M. (2006). Congeneric and (essentially) tau-equivalent estimates of score reliability: What they are and how to use them. Educational and Psychological Measurement, 66(6), 930-944. https://doi.org/10.1177/0013164406288165

Green, S. B., \& Yang, Y. (2015). Evaluation of dimensionality in the assessment of internal consistency reliability: Coefficient alpha and omega coefficients. Educational Measurement: Issues and Practice, 34(4), 14-20. https://doi.org/10.1111/emip.12100

Heppner, W. L., \& Shirk, S. D. (2018). Mindful moments: A review of brief, low-intensity mindfulness meditation and induced mindful states. Social and Personality Psychology Compass, 12(12), e12424. https://doi.org/10.1111/spc3.12424

Herbert, T. B., \& Cohen, S. (1993). Stress and immunity in humans: A meta-analysis review. Psychosomatic Medicine, 55, 364-364.

Hunt, T. (2013). Lambda4: Collection of internal consistency reliability coefficients. https://CRAN.Rproject.org/package=Lambda4

Kaiser, H. F. (1970). A second generation Little Jiffy. Psychometrika, 35(4), 401-415. https://doi.org/10.1007/BF02291817

Kaiser, H. F., \& Rice, J. (1974). Little Jiffy, Mark IV. Educational and Psychological Measurement, 34(1), 111-117. https://doi.org/10.1177/001316447403400115 
Kyriazos, T. A. (2018). Applied psychometrics: Sample size and sample power considerations in factor analysis (EFA, CFA) and SEM in general. Psychology, 09(08), 2207-2230. https://doi.org/10.4236/psych.2018.98126

Lloyd, C., Smith, J., \& Weinger, K. (2005). Stress and diabetes: A review of the links. Diabetes Spectrum, 18(2), 121-127. https://doi.org/10.2337/diaspect.18.2.121

Mars, T. S., \& Abbey, H. (2010). Mindfulness meditation practise as a healthcare intervention: A systematic review. International Journal of Osteopathic Medicine, 13(2), 56-66. https://doi.org/10.1016/j.ijosm.2009.07.005

McCallie, M. S., Blum, C. M., \& Hood, C. J. (2006). Progressive Muscle Relaxation. Journal of Human Behavior in the Social Environment, 13(3), 51-66. https://doi.org/10.1300/J137v13n03_04

Moosbrugger, H., \& Kelava, A. (2012). Testtheorie und Fragebogenkonstruktion (2., aktualisierte und überarbeitete Auflage). Springer Berlin Heidelberg.

Pal, G. K., Ganesh, V., Karthik, S., Nanda, N., \& Pal, P. (2014). The effects of short-term relaxation therapy on indices of heart rate variability and blood pressure in young adults. American Journal of Health Promotion, 29(1), 23-28. https://doi.org/10.4278/ajhp.130131-QUAN-52

Rausch, S. M., Gramling, S. E., \& Auerbach, S. M. (2006). Effects of a single session of large-group meditation and progressive muscle relaxation training on stress reduction, reactivity, and recovery. International Journal of Stress Management, 13(3), 273-290.

https://doi.org/10.1037/1072-5245.13.3.273

Reise, S. P., Bonifay, W. E., \& Haviland, M. G. (2013). Scoring and modeling psychological measures in the presence of multidimensionality. Journal of Personality Assessment, 95(2), 129-140. https://doi.org/10.1080/00223891.2012.725437

Revelle, W. (2020). psych: Procedures for psychological, psychometric, and personality research. Northwestern University. https://CRAN.R-project.org/package=psych

Rosseel, Y. (2012). lavaan: An R package for structural equation modeling. Journal of Statistical Software, 48(2), 1-36. 
Sakakibara, M., Teramoto, Y., \& Tani, I. (2014). Development of a short-form self-report measure to assess relaxation effects. The Japanese Journal of Psychology, 85(3), 284-293. https://doi.org/10.4992/jjpsy.85.13210

Sawhney, G., Jennings, K. S., Britt, T. W., \& Sliter, M. T. (2018). Occupational stress and mental health symptoms: Examining the moderating effect of work recovery strategies in firefighters. Journal of Occupational Health Psychology, 23(3), 443-456.

https://doi.org/10.1037/ocp0000091

Smith, J. C. (2007). Smith Relaxation States Inventory 3 (SRSI3). Lulupress.

Smith, J. C., Wedell, A. B., Kolotylo, C. J., Lewis, J. E., Byers, K. Y., \& Segin, C. M. (2000). ABC relaxation theory and the factor structure of relaxation states, recalled relaxation activities, dispositions, and motivations. Psychological Reports, 86(3_suppl), 1201-1208. https://doi.org/10.2466/pr0.2000.86.3c.1201

Team, R. C. (2020). R: A Language and Environment for Statistical Computing. R Foundation for Statistical Computing. https://www.R-project.org/

Tennant, C. (2002). Life events, stress and depression: A review of recent findings. Australian \& New Zealand Journal of Psychiatry, 36(2), 173-182. https://doi.org/10.1046/j.14401614.2002.01007.x

U.S. Department of Health and Human Services, National Institutes of Health, National Institute of Mental Health. (2019). 5 Things You Should Know About Stress (NIH Publication No. 19-MH8109). https://www.nimh.nih.gov/health/publications/stress/index.shtml

Wadhwa, S. (Ed.). (2017). Stress in the modern world: Understanding science and society. Greenwood.

Zeidan, F., Johnson, S. K., Gordon, N. S., \& Goolkasian, P. (2010). Effects of brief and sham mindfulness meditation on mood and cardiovascular variables. The Journal of Alternative and Complementary Medicine, 16(8), 867-873. https://doi.org/10.1089/acm.2009.0321 


\section{Appendix A}

\section{Original Instructions of the RSQ (in German)}

Im Folgenden finden Sie eine Reihe von Feststellungen. Bitte lesen Sie jede durch und wählen Sie aus den fünf Antworten diejenige aus, die angibt, wie sehr die Feststellung auf $\mathrm{Ihr}$ Befinden jetzt, in diesem Moment, zutrifft. Es gibt keine richtigen oder falschen Antworten. Überlegen Sie bitte nicht lange und lassen Sie keine Frage aus. 


\section{Appendix B}

Table With Bonferroni-Holm Corrected $p$ Values

Table B1

Correlations Between PSQ and RSQ with Bonferroni-Holm Correction

\begin{tabular}{lcc}
\hline \multicolumn{1}{c}{ Correlation } & $p$ value & $\begin{array}{c}\text { Significance level after } \\
\text { correction }\end{array}$ \\
\hline Tension \& cardiovascular score & .03044 & .05 \\
Joy \& general relaxation score & .01676 & .025 \\
Joy \& cardiovascular score & .01194 & .01667 \\
Joy \& muscle score & .008106 & .0125 \\
Tension \& general relaxation score & .0006075 & .01 \\
Tension \& muscle score & .0000107 & .008333 \\
Joy \& sleepiness score & .000004298 & .00714 \\
Tension \& sleepiness score & .000002403 & .00625
\end{tabular}

Note. Correlations were ordered by $p$ value, then for every correlation the new significance level was computed by the formula given by Eid et al. $(2017$, p. 418$)$ with $(\alpha=.05)$. All correlations remained significant. 